\title{
Cost-effectiveness of cognitive-behavioural therapy as an adjunct to pharmacotherapy for treatment-resistant depression in primary care: economic evaluation of the CoBalT Trial
}

Sandra Hollinghurst, Fran E. Carroll, Anna Abel, John Campbell, Anne Garland, Bill Jerrom, David Kessler, Willem Kuyken, Jill Morrison, Nicola Ridgway, Laura Thomas, Katrina Turner, Chris Williams, Tim J. Peters, Glyn Lewis and Nicola Wiles

\section{Background}

Depression is expensive to treat, but providing ineffective treatment is more expensive. Such is the case for many patients who do not respond to antidepressant medication.

\section{Aims}

To assess the cost-effectiveness of cognitive-behavioural therapy (CBT) plus usual care for primary care patients with treatment-resistant depression compared with usual care alone.

\section{Method}

Economic evaluation at 12 months alongside a randomised controlled trial. Cost-effectiveness assessed using a cost-consequences framework comparing cost to the health and social care provider, patients and society, with a range of outcomes. Cost-utility analysis comparing health and social care costs with quality-adjusted life-years (QALYS).

\section{Results}

The mean cost of CBT per participant was $\mathrm{f910}$. The difference in QALY gain between the groups was 0.057, equivalent to 21 days a year of good health. The incremental cost-effectiveness ratio was $£ 14911$ (representing a $74 \%$ probability of the intervention being cost-effective at the National Institute of Health and Care Excellence threshold of f20000 per QALY). Loss of earnings and productivity costs were substantial but there was no evidence of a difference between intervention and control groups.

\section{Conclusions}

The addition of CBT to usual care is cost-effective in patients who have not responded to antidepressants. Primary care physicians should therefore be encouraged to refer such individuals for CBT.

\section{Declaration of interest}

C.W. and A.G. are CBT workshop leaders. W.K. teaches nationally and internationally on CBT.
Depression is a major cause of morbidity and disease burden worldwide. ${ }^{1}$ It is also costly. An estimated $£ 1.7$ billion was spent in England in 2007 treating patients with depression, ${ }^{2}$ an amount that has risen every year as prevalence has increased and more treatments have been made available. However, these costs are dwarfed by the wider societal costs of lost productivity, which have been estimated to be more than four times greater than the direct health service costs. ${ }^{2}$ This means that although treating patients with depression is expensive it is even more expensive not to treat them or to provide ineffective treatment. This is the case with a third of patients with clinical depression treated with antidepressant medication: ${ }^{3}$ this is the proportion who do not respond to treatment. It has, indeed, been shown that the financial impact of treatment-resistant depression is considerable $e^{4-7}$ in addition to the level of psychological suffering and risks involved with the disorder. There is therefore an urgent need to identify alternative cost-effective methods of treatment for this patient group. Cognitive-behavioural therapy (CBT), delivered in different ways (for example individual face to face, group therapy, computerised packages), is cost-effective in a range of conditions ${ }^{8-10}$ including previously untreated depression, ${ }^{11,12}$ and in the parent trial of this study ${ }^{13,14}$ it has been shown to be beneficial to patients with treatment-resistant depression. The aim of this study was to assess the cost-effectiveness of individual face-to-face CBT as an adjunct to usual care (which included pharmacotherapy) for primary care patients with treatment-resistant depression compared with usual care alone.

\section{Method}

\section{Study design}

We carried out an economic evaluation alongside a randomised controlled trial (RCT) to establish the cost-effectiveness, over 12 months, of CBT in addition to usual care for primary care patients with treatment-resistant depression. The trial and its clinical findings have been reported in full elsewhere. ${ }^{13,14}$ The main perspective for the economic evaluation was the health and social care provider. As previous studies suggest that personal costs incurred by patients and productivity costs as a result of time off work might also be important, ${ }^{7,11}$ these were assessed and reported separately. We carried out two types of evaluation: a cost-consequences analysis reporting cost from each of the three perspectives (health and social care, patients and lost productivity) alongside a range of outcomes; and a cost-utility analysis in line with recommendations of the National Institute for Health and Care Excellence (NICE) $)^{15}$ comparing cost from the National Health Service (NHS) and Personal Social Services (PSS) perspective (with quality adjusted life years, QALYs).

\section{Setting and participants}

We recruited UK-based primary care patients aged between 18 and 75 years who had adhered to antidepressant medication for at least 6 weeks but who continued to have significant depressive symptoms. 
Entry criteria were based on a Beck Depression Inventory-II $(\mathrm{BDI}-\mathrm{II})^{16}$ score of 14 or more and an ICD-10 diagnosis of depression using the Revised Clinical Interview Schedule (CIS-R). ${ }^{17,18}$ Recruitment took place between November 2008 and October 2010 in Bristol, Exeter and Glasgow.

\section{Intervention and usual care}

Patients randomised to receive CBT in addition to usual care were offered between 12 and 18 sessions each lasting about an hour, which took place at their general practitioner (GP) surgery or a similar location. All 11 therapists, working across the 3 sites, used the same CBT manuals for depression, ${ }^{19,20}$ informed by Moore \& Garland's manual on chronic depression, ${ }^{21}$ and received weekly clinical supervision. Therapists were flexible in responding to problems raised by the patient, for example, by targeting symptoms of anxiety using appropriate cognitive-behavioural models, if these were considered important. Emphasis was also given to formulating the psychopathology in terms of conditional beliefs.

Patients randomised to the control group continued to receive unrestricted usual care from their GP, including antidepressant treatment as judged appropriate by the patients' GP or a referral as required. At the time of randomisation all patients in both groups were taking antidepressants.

\section{Outcome measures}

The primary outcome for the trial was a reduction of at least $50 \%$ in the BDI-II score at 6 months compared with baseline. In addition, a range of outcomes were recorded at 12 months to assess the sustainability of any effect. These included BDI-II score, remission (BDI-II $<10$ ), the 12-item Short Form (SF-12) mental and physical subscales ${ }^{22}$ and the EQ-5D-3L. ${ }^{23}$ All outcomes were measured at 6 and 12 months and, where possible, this was carried out face to face at an appointment with a researcher.

\section{Resource use}

We measured all health and social care, personal out-of-pocket expenditure such as travel costs and use of private therapies and over-the-counter medications, and time off work over the 12 -month period. Information about the use of primary care and prescribed medication was extracted, with consent, from GP records. We included all primary and community care irrespective of the reason for the encounter because the nature of primary care makes it difficult to identify consultations that are for underlying mental ill health. ${ }^{24}$ In the case of hospital use, where access is controlled by GPs acting as gatekeepers, we can be more confident of identifying mental health-related use. We used the information provided by the participants about who they had seen (for example psychiatrist, psychiatric nurse, psychologist), the reason for the visit (for example, 'overdose', 'panic attack', 'self-harm') and/or the name of the ward, clinic or facility to identify which appointments and overnight stays to include. We included all aspects of mental health, not just depression, to avoid excluding potentially important encounters.

A questionnaire was administered to all trial participants at 6-monthly intervals in which they were asked for information about resource use related to their mental health during the 6-month period that could not be obtained from GP records. This included detailed questions about the use of hospital services to supplement that on record, and information about use of social care. We also asked about out-of-pocket expenditure including: use of private healthcare, therapies and self-help groups; over-the-counter medication; travel to GP, CBT sessions and hospital; use of informal care and the voluntary sector; and time off work.

The cost of the intervention was estimated using information recorded by the trial therapists about the number and length of CBT sessions booked and attended by each participant.

\section{Valuation of resource use}

Online Table DS1 gives the unit costs and sources used to value the healthcare resources. We used Curtis ${ }^{25}$ to value primary and community health and social care wherever an estimate was available. Use of walk-in centres, NHS Direct (in England) and NHS 24 (in Scotland) was valued using national evaluations ${ }^{26,27}$ and for out-of-hours care we obtained estimates directly from a local provider (Brisdoc Healthcare Services). Department of Health reference costs ${ }^{28}$ were used to value all hospital-based care and the British National Formulary (BNF) ${ }^{29}$ was used for prescribed medication. Personal expenditure was reported directly by the participants, the exception being travel by car, which was reported as mileage and costed using the Automobile Association (AA) schedule of motoring costs. ${ }^{30}$ Time off work was valued using the median gross weekly earnings by age and gender. ${ }^{31}$

Most of the therapists in the trial were appointed at Band $8 \mathrm{a}$, which is about $£ 73$ per hour face-to-face time allowing for oncosts (National Insurance, superannuation), overheads, and non-contact time of $50 \% .{ }^{25}$ The first CBT session was 90 min in length with subsequent sessions lasting $60 \mathrm{~min}$. Appointments that were cancelled were not included in the costing, but when a patient did not cancel and failed to attend we included an amount equal to half the usual rate, reflecting the fact that therapists would make some use of the time but would not be fully productive. Supervision was generally carried out in groups of two or three. Therapist time for this was subsumed in the non-contact time but we included an estimate of $\mathfrak{E} 2.50$ per hour of therapy delivered to cover the cost of the supervisors' time. This was based on the salary of a consultant psychiatrist/consultant clinical psychologist. All costs were valued in pound sterling at 2010 prices, adjusted for inflation where necessary. ${ }^{25}$

\section{Data analysis}

We investigated the amount of each resource used by participants in both groups using frequencies, means and medians. Mean total cost per participant was derived by combining resource use with unit costs. In line with the recommendations of NICE, ${ }^{15}$ utility values were derived from responses to the EQ-5D-3L at baseline, 6 months and 12 months using valuations from the UK general population. ${ }^{32}$ These values, representing health-related quality of life on a scale between zero (death) and one (best imaginable health), were used to compute QALYs using the area under the curve approach and adjusting for any difference between the groups at baseline. ${ }^{33}$

Using all available data, a cost-consequences matrix was constructed to compare, by group: mean cost per participant from each perspective; clinical outcomes based on the BDI-II; quality of life using the SF-12; and QALYs. We used the multiple imputation by chained equation procedure to address the issue of missing cost and QALY data. ${ }^{34}$ This method uses regression techniques to estimate missing values, based on the values of available data. The 'ice' command (version 1.9.5 PR/IW 15apr2011) in Stata v12 on Windows was used to generate five data-sets using ten switching procedures; in addition to a range of cost and EQ-5D-3L variables the model also included randomisation group, age and gender.

An incremental cost-effectiveness ratio (ICER) was estimated by dividing the difference in mean cost between the groups by 
the mean difference in QALYs. Uncertainty in the estimates of cost and QALYs was captured using standard deviations around the point estimates and confidence intervals around incremental differences. Uncertainty around the ICER was captured using non-parametric bootstrapping. Five thousand replicates of the ICER were generated and these were used to estimate: (a) confidence intervals around the net monetary benefit; and (b) a cost-effectiveness acceptability curve. Discounting was not carried out because the analysis was restricted to costs and outcomes over 1 year. All analyses were conducted using Microsoft Excel and Stata 12.1.33.

\section{Sensitivity analyses}

We performed a series of four one-way sensitivity analyses on the cost per QALY results to address areas of uncertainty in our assumptions. Two of these addressed cost, one addressed QALYs and one both cost and QALYs.

First, we investigated the importance of the grade of therapist. In the trial the therapists were mainly Band $8 \mathrm{a}$ and costed as such although in practice they are often appointed at a lower grade. We estimated the effect on cost per QALY if all therapists were instead costed at Band 7, as is often the case in Improving Access to Psychological Therapies (IAPT) ${ }^{35}$ 'high intensity' and other CBT services across the UK. Second, the patients in this trial were all recruited from primary care and this is where they are largely cared for. Secondary care is relatively uncommon but expensive and can affect results disproportionately; because of the small numbers involved, hospital use may not be balanced between the groups simply by chance and results could be misleading. We removed hospital costs from the analysis to see whether this was the case. Our third area of sensitivity analysis was around deriving utility weights for the calculation of QALYs. In the main analysis we followed NICE recommendations and used the EQ-5D, but as the trial also collected data on the SF-12, and because of known concerns around the sensitivity of the
EQ-5D-3L in depression, ${ }^{36}$ we added four questions from the 36-item Short Form (SF-36) to the questionnaire to enable the Short Form - six dimensions (SF-6D) algorithm ${ }^{37}$ to be used to calculate QALYs using this instrument. Finally, we investigated the effect of imputing missing data by comparing the imputed results with those restricted to complete cases, that is, including only those participants for whom we had complete cost and QALY data at both 6 and 12 months.

\section{Results}

A total of 469 patients were recruited to the trial, 234 to CBT plus usual care and 235 to usual care alone. The majority (72\%) were women and the mean age was 49.6 years. The mean BDI-II score at baseline was 31.8 (scores of 29 and above are interpreted as 'severe') and $70 \%$ had been on current antidepressant medication for more than 12 months. Nearly all participants (97\%) gave permission to access their GP notes to obtain data about primary care encounters and prescribed medication. The response rate to the questionnaire was high: $83 \%$ of participants in both groups returned the questionnaire at both 6 and 12 months, although not all participants completed all sections at both time points. We had complete NHS and PSS cost and QALY data for 368 (78\%) of participants and complete personal cost data for 274 (58\%).

\section{Resource use}

Table 1 gives an indication of the range and level of resources used over the 12 months. We show frequencies, means and medians for each category of resource for all participants for whom we had data. Denominators therefore vary by category. Most participants (89\%) saw their GP at least once during the year and on average they had about eight consultations. Over $90 \%$ of patients had at least one prescription for an antidepressant and at least 10\% in both groups had more than 15. Patients in the usual care group

\begin{tabular}{|c|c|c|c|c|c|c|}
\hline & \multicolumn{3}{|c|}{ Intervention } & \multicolumn{3}{|c|}{ Usual care } \\
\hline & $n$ & $\begin{array}{l}\text { Number of } \\
\text { encounters } \\
\text { Mean (s.d.) }\end{array}$ & $\begin{array}{c}\text { Accessing } \\
\text { the service } \\
n(\%)\end{array}$ & $n$ & $\begin{array}{l}\text { Number of } \\
\text { encounters } \\
\text { Mean (s.d.) }\end{array}$ & $\begin{array}{c}\text { Accessing } \\
\text { the service } \\
n(\%)\end{array}$ \\
\hline General practitioner consultations & 229 & $7.6(7.0)$ & $204(89)$ & 224 & $8.3(6.2)$ & $200(89)$ \\
\hline Nurse consultations & 229 & $2.0(4.1)$ & $129(56)$ & 224 & $2.0(2.8)$ & $125(56)$ \\
\hline Other surgery-based consultations & 229 & $0.9(2.2)$ & $83(36)$ & 224 & $1.2(2.6)$ & $88(39)$ \\
\hline Out of hours, walk-in centres and NHS Direct & 188 & $0.2(0.7)$ & $24(13)$ & 182 & $0.2(0.8)$ & $19(10)$ \\
\hline Exercise on referral & 191 & $0.7(3.8)$ & $11(6)$ & 194 & $1.3(7.9)$ & $10(5)$ \\
\hline Alternative therapy & 191 & $0.8(3.5)$ & $21(11)$ & 194 & $1.0(4.0)$ & $21(11)$ \\
\hline CBT and CCBT & 195 & $1.3(4.2)$ & $14(7)$ & 195 & $2.0(5.5)$ & $23(12)$ \\
\hline Antidepressant medication & 229 & $9.0(6.8)$ & $205(90)$ & 224 & $8.7(5.9)$ & $205(92)$ \\
\hline Other prescribed medication & 229 & $28.4(36.8)$ & $190(83)$ & 224 & $34.0(43.3)$ & $199(89)$ \\
\hline Accident and emergency visits & 192 & $0.1(0.3)$ & $7(4)$ & 192 & $0.0(0.2)$ & $8(4)$ \\
\hline Out-patient visits & 192 & $0.1(0.5)$ & $13(7)$ & 194 & $0.1(0.3)$ & $11(6)$ \\
\hline In-patient stays & 192 & $0.0(0.2)$ & $3(2)$ & 192 & $0.0(0.2)$ & $2(1)$ \\
\hline Trial CBT & 234 & $11.2(6.3)$ & $214(91)$ & & & \\
\hline Social worker visits & 191 & $0.0(0.4)$ & $3(2)$ & 195 & $0.0(0.3)$ & $5(3)$ \\
\hline Home help & 192 & $0.5(4.7)$ & $3(2)$ & 194 & $1.8(16.2)$ & $4(2)$ \\
\hline Self-help groups & 192 & $0.2(1.9)$ & $3(2)$ & 194 & $0.4(3.5)$ & $4(2)$ \\
\hline Day centre attendance & 192 & $0.6(3.7)$ & $6(3)$ & 194 & $0.4(5.6)$ & $3(2)$ \\
\hline Number of days off work & 182 & $12.0(44.8)$ & $40(22)$ & 185 & $12.1(38.4)$ & $42(23)$ \\
\hline
\end{tabular}




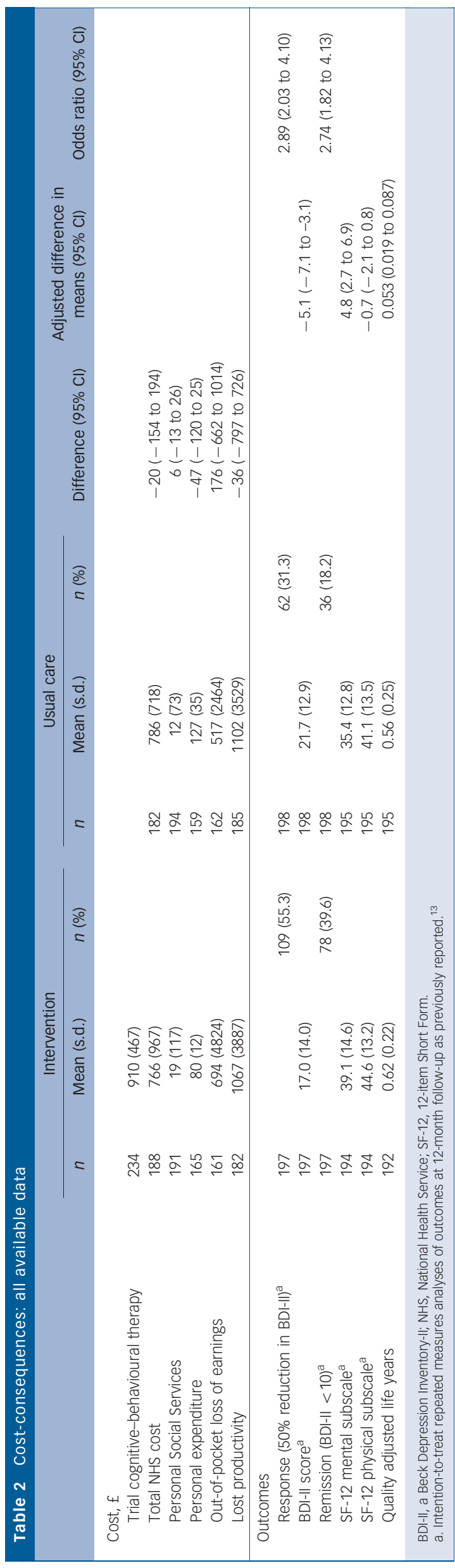

had more 'other' (non-antidepressant) medications prescribed than those in the intervention group, although variation was high with the number of prescriptions ranging from 0 to over 150 in both groups. Use of hospital services was low: 15 (4\%) of participants went to accident and emergency, $24(6 \%)$ attended at least one out-patient clinic and $5(1 \%)$ had an overnight stay. Seventy-five (16\%) participants reported using at least one session of computerised CBT (cCBT) or talking therapy (for example, counselling, CBT) outwith the trial therapy; overall this was more common in the usual care group (41 v. 34) although more intervention participants accessed cCBT (11 v. 7).

Just under a quarter $(22 \%)$ of participants reported having some time off work because of their illness, and for these the mean number of days off work was 54 . A small number $(7,2 \%)$ reported they were unable to work at all during the trial period. There was no difference between the groups. Most $(138,59 \%)$ participants in the intervention group received between 12 and 18 sessions of $\mathrm{CBT}$ as per protocol although a small number $(20,8.5 \%)$ received no therapy.

\section{Cost consequences}

In Table 2 we show mean cost and outcome per participant, by group, for each category of cost, and a range of primary and secondary outcomes. These estimates use all available data, which gives a different number of observations for each category. The total cost of a course of 18 sessions was $£ 1400$. Allowing for those participants who had less than 18 sessions, the mean cost per session of CBT delivered was $\mathfrak{E} 81$, which equates to $£ 910$ per randomised participant.

The mean cost, per patient, of caring for those in the usual care group was slightly higher than for the intervention group, mainly because of more visits to the GP and more non-antidepressant medication. Out-of-pocket personal expenses were also higher because of greater expenditure on alternative therapies and so too was the value of time off work (lost productivity). However, the wide confidence intervals around all these categories of cost reflect considerable variation and there is no conclusive evidence of a difference between the two groups.

All three clinical outcomes based on the BDI-II indicate that patients in the intervention group improved more than those receiving usual care alone. As reported previously, ${ }^{14}$ the mean score at 12 months was 5.1 points lower for these patients and twice as many in this group recorded a 50\% reduction in BDI-II compared with those receiving usual care.

\section{Cost-utility analysis}

The base case cost-utility analysis is presented in Table 3. These results are from the NHS and PSS perspective and include imputed missing cost and QALY data. The cost of the intervention (£910) is slightly offset by the higher cost (£59) of health and social care in the usual care group giving an incremental cost of \&850. In line with the clinical outcomes, participants receiving the intervention experienced a better health-related quality of life as measured by QALYs (0.61 v. 0.55), giving a cost per QALY over the 12 months of $£ 14911$.

If society is willing to pay $£ 20000$ per QALY, as suggested by NICE, ${ }^{15}$ the net monetary benefit per patient per year is $£ 289$ (95\% CI $-\mathfrak{E} 603$ to $\mathfrak{E} 1182$ ) and the probability that the intervention is cost-effective is 0.74 (Fig. 1). These rise to $\mathfrak{E} 859$ (95\% CI $-£ 455$ to $£ 2179$ ) and 0.91 at a threshold of $£ 30000$ per QALY.

\section{Sensitivity analyses}

The results of the four one-way sensitivity analyses are shown in Table 4 and Fig. 2. If therapists were employed at Band 7 the 


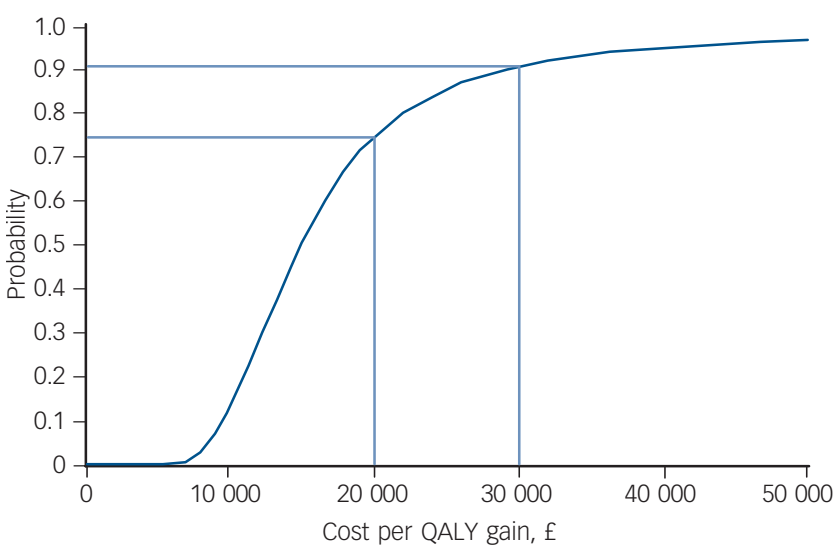

Fig. 1 Cost-effectiveness acceptability curve showing the probability that the intervention is cost-effective at different levels of willingness to pay. QALY, quality adjusted life year.

ICER would be lower at $\mathfrak{E} 13006$. Hospital costs were similar in the two groups so excluding these makes very little difference to the cost per QALY. The third sensitivity analysis, using data from the SF-12 to estimate QALYs, gives slightly higher estimates for both groups than using the EQ-5D-3L and the difference between them is narrowed, which results in a higher ICER of $£ 29626$. Finally, we tested the implication of using imputed data by looking at results for complete cases - those for whom we had data on cost and QALYs at both 6 and 12 months. These participants had more therapy sessions than the average (12.8 v. 11.2), at a mean cost of $£ 1020$. Other costs and QALYs remain broadly the same, giving an ICER of $£ 18361$ for those with complete data.

\section{Discussion}

\section{Summary of main findings}

From the perspective of a healthcare provider, CBT as an adjunct to usual care (that includes pharmacotherapy) is a cost-effective treatment for primary care patients with treatment-resistant depression over a 12-month period. Out-of-pocket personal expenditure was dominated by loss of earnings, and the value of lost productivity was substantial, but neither of these differed between the two groups.

\section{Strengths and limitations of the study}

This economic evaluation was part of a multidisciplinary study involving a large multicentre RCT. The trial benefited from a pilot study, ${ }^{38}$ recruited to target and achieved impressive follow-up rates. Delivery of the CBT intervention was evaluated by an independent assessor, ensuring consistency and generalisability.

As with all economic evaluations there were some missing data. However, the largest component of cost was the CBT intervention, for which we had complete data on all participants, and $90 \%$ of other health and social care costs were based on data retrieved from primary care notes (primary care consultations and prescribed medication), which were $97 \%$ complete. The QALY data were $83 \%$ complete. For this reason we feel confident in presenting, as our base case scenario, the cost-utility analysis with the small amount of missing data imputed. Our sensitivity analysis, using complete cases, reinforces this decision. The results imply the cost of health and social care, and QALYs, are unaffected by the imputation. The ICER is affected by the cost of the intervention for the subset of participants who provided complete data; these participants engaged more fully with the trial, which also meant attending more sessions of therapy than those for whom we did not have complete data.

Data on personal costs and time off work because of mental health problems were less complete. Although overall questionnaire response rates were high $(83 \%)$, not all participants completed all sections at both 6 and 12 months. Furthermore, we acknowledge that 6 months is a long period of recall for patients, particularly those with mental health problems, and we cannot be certain of the accuracy of these data. For these reasons we present these costs in a cost-consequences format, using all available data by category, to preserve transparency. Comparison between the groups is valid because completion rates were similar and we have no reason to believe that accuracy of recall would be any different between the two groups.

The estimates of QALYs using the SF-6D are substantially higher than those using the EQ-5D-3L and the difference between the groups is smaller - although the variability is lower. The difference of 0.03 (95\% CI $0.057-0.029$ ) of a QALY equates to 11 days in perfect health, which is a useful health gain. A number of studies have looked into the importance of differences between these two measures of health-related quality of life in different populations ${ }^{39,40-42}$ but there is limited evidence about patients with moderate to severe depression. In a study of patients with

Table 3 Cost-utility analysis - National Health Service (NHS) and Personal Social Services (PSS) perspective

\begin{tabular}{|c|c|c|}
\hline & Intervention $(n=234)$ & Usual care $(n=235)$ \\
\hline All primary care, f: mean (s.d.) & $267(285)$ & $294(241)$ \\
\hline Prescribed medication, f: mean (s.d.) & $352(614)$ & $418(558)$ \\
\hline Hospital care, f: mean (s.d.) & $64(323)$ & $38(128)$ \\
\hline PSS, f: mean (s.d.) & $20(107)$ & $14(67)$ \\
\hline NHS and PSS services, f: mean (s.d.) & 704 (938) & $763(697)$ \\
\hline Cost of CBT, f: mean (s.d.) & $910(467)$ & - \\
\hline Total cost NHS and PSS perspective, f: mean (s.d.) & $1614(1100)$ & $763(697)$ \\
\hline QALY, mean (s.d.) & $0.608(0.22)$ & $0.551(0.24)$ \\
\hline Incremental cost, $£(95 \% \mathrm{Cl})$ & 850 (683 to 1017) & \\
\hline Incremental benefit, QALY gain $(95 \% \mathrm{CI})$ & 0.057 (0.015 to 0.099$)$ & \\
\hline Incremental cost-effectiveness ratio, f: cost per QALY gain & 14911 & \\
\hline $\begin{array}{l}\text { Median net monetary benefit, } £(P(\text { net monetary benefit }>0)) \\
\text { Willingness to pay }(\lambda)=£ 20000 \text { per QALY } \\
\text { Willingness to pay }(\lambda)=£ 30000 \text { per QALY }\end{array}$ & $\begin{array}{l}289(0.74) \\
859(0.91)\end{array}$ & \\
\hline
\end{tabular}




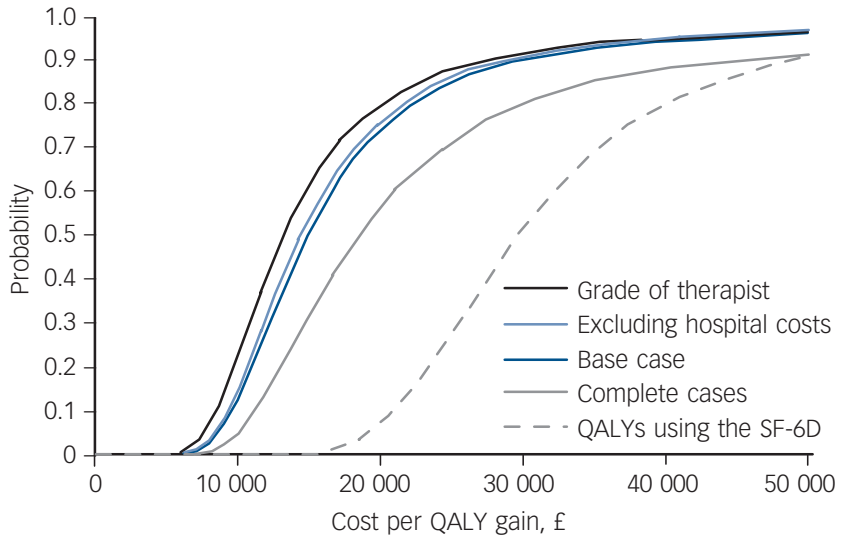

Fig. 2 Cost-effectiveness acceptability curves for scenarios explored in the sensitivity analysis.

QALY, quality adjusted life year; SF-6D, Short Form - six dimensions. wide-ranging mental health problems Lamers et al found, as we did, that the EQ-5D resulted in larger health gains than the SF-6D, over a 12 -month period. ${ }^{43}$ This was especially true for patients with the most severe conditions. Our base case analysis follows the NICE guidance in using the EQ-5D, ${ }^{15}$ a view reiterated in a recent review of low-intensity psychological interventions for the secondary prevention of relapse after depression. ${ }^{44}$

The incremental benefit found using the SF-6D is somewhat lower than that using the EQ-5D-3L and the estimated ICER of $£ 29626$ is considerably higher. Although this is just below the upper NICE threshold of $£ 30000$, it must be noted that recent research into a revised threshold suggests that in the UK the 'true' value of a QALY is nearer $£ 18000 .{ }^{45}$ If this is the case, the result of this sensitivity analysis casts some doubt over the robustness of our base case findings, suggesting that more investigation into the most appropriate measure for obtaining QALYs in this context would be useful.

\section{Comparison with existing literature}

A few studies have estimated the relative cost burden of caring for patients with treatment-resistant depression and, unsurprisingly, there is general agreement that this patient population uses more healthcare resources than many others. ${ }^{4-6}$ The patients in our study attended their GP more frequently than average ${ }^{46}$ and had more antidepressant and other prescribed medications than reported in other studies. Use of these resources was slightly lower in the patients receiving CBT (and who, on average, improved most during the year) but the difference was quite small. This is similar to previous findings in trials of primary care patients with a new episode of depression. ${ }^{12,47}$ Mukuria et al ${ }^{48}$ found very similar estimates of cost per QALY in their evaluation of IAPT services in one part of the UK despite differences in the patient group and the intervention. However, uncertainty around their estimates was larger than in our study, leading to a somewhat more cautious conclusion.

The value of lost productivity because of mental illness is a major concern. It is also a contentious area in terms of how best to measure and value time off work because of illness. In this study we used the most common method - the 'human capital' approach, valuing all time off work using published rates of pay. The employment status of patients in this study varied hugely, with only a quarter reporting time off work; for those who did, their absence was on average about 10 weeks. This is shorter than the 19 weeks reported for the 6-month follow-up of the THREAD study, ${ }^{47}$ although it may be accounted for by the fact that a number $(27 \%)$ of our participants reported that they were 'unemployed due to ill health'. As we had no detailed information about the circumstances of these participants, and whether this was because of depression, we did not include them in our estimates of productivity losses. Consequently, our estimated cost of just over $£ 1000$ per participant over the year appears modest. It

Table 4 Sensitivity analyses - National Health Service (NHS) and Personal Social Services (PSS) costs and quality adjusted life years (QALYS)

\begin{tabular}{|c|c|c|c|}
\hline & Intervention & Usual care & Difference $(95 \% \mathrm{Cl})$ \\
\hline $\begin{array}{l}\text { Base case } \\
\text { Cost, } f \text { : mean (s.d.) } \\
\text { QALYS, mean (s.d.) } \\
\text { ICER, } f \\
\text { Median NMB, } f(P(N M B>0)) \text { at } \lambda=f 20000\end{array}$ & $\begin{array}{l}1614(1100) \\
0.608(0.22)\end{array}$ & $\begin{array}{l}763 \text { (697) } \\
0.551(0.24)\end{array}$ & $\begin{array}{l}850(683-1017) \\
0.057(0.015-0.099) \\
14911 \\
289(0.74)\end{array}$ \\
\hline $\begin{array}{l}\text { Grade of therapist } \\
\text { Cost, } f \text { : mean (s.d.) } \\
\text { ICER, } f \\
\text { Median NMB, } f(P(N M B>0)) \text { at } \lambda=£ 20000\end{array}$ & 1505 (1071) & 763 (697) & $\begin{array}{c}742(578-905) \\
13006 \\
391(0.80) \\
\end{array}$ \\
\hline $\begin{array}{l}\text { Excluding hospital costs } \\
\text { Cost, } f: \text { mean (s.d.) } \\
\text { ICER, } f \\
\text { Median NMB, } f(P(N M B>0)) \text { at } \lambda=£ 20000\end{array}$ & 1550 (1003) & 726 (681) & $\begin{array}{l}824(669-980) \\
14453 \\
326(0.76)\end{array}$ \\
\hline $\begin{array}{l}\text { QALYS using the SF-6D } \\
\text { QALYS } \\
\text { ICER } \\
\text { Median NMB, } f(P(N M B>0)) \text { at } \lambda=£ 20000\end{array}$ & $0.613(0.09)$ & $0.584(0.09)$ & $\begin{array}{l}0.029(0.014-0.043) \\
29626 \\
-274(0.08)\end{array}$ \\
\hline $\begin{array}{l}\text { Completed cases } \\
\text { Cost, } f \text { : mean (s.d.) } \\
\text { QALYs, mean (s.d.) } \\
\text { ICER, } f \\
\text { Median NMB, } f(P(N M B>0)) \text { at } \lambda=£ 20000\end{array}$ & $\begin{array}{l}1810(1119) \\
0.614(0.24)\end{array}$ & $\begin{array}{l}799(725) \\
0.559(0.24)\end{array}$ & $\begin{array}{c}1011(817-12304) \\
0.055(0.202-0.090) \\
18361 \\
82(0.57)\end{array}$ \\
\hline
\end{tabular}


is also somewhat lower than that found in studies in the USA, where much of this work has taken place., ${ }^{7,49}$

\section{Implications for clinicians, patients and policy makers}

Overall, the findings from the cost-consequences and cost-utility analyses suggest that CBT should be considered in addition to medication as a treatment for primary care patients with depression who have not responded to at least 6 weeks' treatment with antidepressant medication. This would increase demand on CBT and therefore necessitate more training of therapists. Despite the IAPT initiative there is a still a long waiting list for 'high intensity' CBT and, because of the nature of the illness, patients with depression are often reluctant to ask for psychological treatments. The usual care arm in this trial is an example of current policy; there is unmet need in the UK and, probably even more so, internationally where no IAPT initiative exists. ${ }^{50}$

\section{Unanswered questions and future research}

Not all participants in the trial intervention group completed the course of CBT. Our analysis, based on intention to treat, includes all participants irrespective of the amount of therapy they received. If reasons for non-adherence were explored and addressed in an implementation programme, the costs and benefits could be affected and the extent of this is unknown.

The results presented in this study are restricted to the 12-month follow-up period of the trial, which is an appropriate timescale given the mean time to complete the intervention was just over 6 months. However, it would be valuable to know how effective and cost-effective this type of therapy is for this patient group over the long term, from the perspective of a healthcare provider, and for society in general. An important aim of CBT is to help patients manage their own problems by incorporating therapeutic strategies into their everyday activities. Although we acknowledge the limited follow-up period of the trial, it seems reasonable to suggest that patients in the treatment group may be better equipped to adopt these strategies, and may thus potentially have better health in the future. In addition, mental health problems are an important cause of absence from work. ${ }^{51}$ In this present study, there was no difference in time off work between the two groups. However, if the therapy has a lasting effect, this may not be the case over a longer period of time, and one might reasonably speculate on the possibility of improved work attendance among individuals receiving the CBT intervention. A study of longer-term costs and benefits is thus necessary, and could incorporate a decision analytic model; it is crucial that evidence is obtained to both substantiate and quantify this potential for long-term gain. If such an intervention was found to be cost-effective over the long term, this would have significant implications for recommendations as to how depression should be managed.

The IAPT initiative in the UK has led to increased investment for psychotherapy including CBT. The referral rate to this service has been increasing yet their own assessment suggests that only $2.4 \%$ of potentially eligible individuals, estimated from the psychiatric morbidity surveys, were referred to IAPT in 2012. ${ }^{35}$ It is likely there is a considerable unmet need for CBT in those who have not responded to antidepressants, even in the UK following its IAPT initiative. Whether CBT is 'affordable' depends on the prevalence of the condition, and on the demand. Many people with treatment-resistant depression do not want psychological treatments. Nevertheless, our findings justify recommending further investment in 'high intensity' CBT lasting 12-18 sessions.
In conclusion, CBT when added to usual care is a cost-effective treatment for patients with treatment-resistant depression. Investment in CBT services for patients who have not responded to antidepressants would represent an efficient use of healthcare resources and would offer the potential to substantively alleviate the burden of disease.

\begin{abstract}
Sandra Hollinghurst, BA, MA, PhD, Fran E. Carroll, BSC, MSC, PhD, School of Social and Community Medicine, University of Bristol, Bristol; Anna Abel, BSC, Mphil; John Campbell, MD, FRCGP, University of Exeter Medical School, Exeter; Anne Garland, MSC, Nottingham Psychotherapy Unit, Nottinghamshire Healthcare NHS

Trust, Nottingham; Bill Jerrom, PhD, Avon and Wiltshire Mental Health Partnership Trust, Nottingham; Bill Jerrom, PhD, Avon and Wittshire Mental Health Partners
NHS Trust, Chippenham; David Kessler, MD, School of Social and Community Medicine, University of Bristol, Bristol; Willem Kuyken, BSc, PhD, DclinPsy, School of Psychology, University of Exeter, Exeter; Jill Morrison, MBChB, MSC, PhD, Academic Unit of General Practice and Primary Care, Institute of Health and Wellbeing, University of Glasgow, Glasgow; Nicola Ridgway, MA, PgDip, PhD, Academic Unit of Mental Health and Wellbeing, Institute of Health and Wellbeing, University of Glasgow, Gartnavel Royal Hospital, Glasgow; Laura Thomas, BA, MPhil, Katrina Turner, BSc, MSc, PhD, School of Social and Community Medicine, University of Bristol, Bristol; Chris Williams, MBChB, BSC, MmedSc, MD, Academic Unit of Mental Health and Wellbeing, Institute of Health and Wellbeing, University of Glasgow, Gartnavel Royal Hospital, Glasgow; Tim J. Peters, BSC, MSC, PhD, School of Clinical Sciences, University of Bristol, Bristol; Glyn Lewis, PhD, FRCPsych, Nicola Wiles, BSC, PhD, School of Social and Community Medicine, University of Bristol, Bristol, UK
\end{abstract}

Correspondence: Sandra Hollinghurst, BA, MA, PhD, Department of Social and Community Medicine, University of Bristol, Canynge Hall, Whatley Road, Bristol BS8 2PS, UK. Email s.p.hollinghurst@bristol.ac.uk

First received 18 Dec 2012, final revision 3 May 2013, accepted 18 Jul 2013

\section{Funding}

This research was funded by the National Institute for Health Research Health Technology Assessment (NIHR HTA) programme (project number: 06/404/02).

\section{Acknowledgements}

We are grateful to all the patients, practitioners and GP surgery staff who took part in this research. We would like to thank the members of our Trial Steering Committee and Data Monitoring Committee for their valuable advice and support during the project. We acknowledge the additional support that has been provided by the Mental Health Research Network (MHRN), Scottish Mental Health Research Network (SMHRN), Primary Care Research Network (PCRN) and Scottish Primary Care Research Network (SPCRN). Finally, we would also like to thank the following colleagues who have contributed to the CoBalT study, through recruitment and retention of patients, provision of administrative support, or delivery/supervision of therapy: Joy Farrimond, Mary Yarwood, Katrina Crook, Caroline Baker, Nathan Filer, Alex Burrage, Samantha Green, Meyrem Musa, Emma Riggs, Ros Fortune and Kate Chapman in Bristol; Rachel Winder, Caroline Jenkinson, Alice Garood, Holly Sugg, Miriam Cassell, Rob Kidney and Clare Bootle in Exeter; and Monica Cairns, Seonaid Cleare, Janice Reid, June Anderson, Jacqueline McTaggart, Katy Park, Eileen Riddoch and Catriona Kent in Glasgow. Finally, we would like to acknowledge the contribution of Debbie Sharp who was a co-applicant on the original grant application.

\section{References}

1 World Health Organization. The Global Burden of Disease: 2004 Update. WHO, 2008.

2 National Institute for Health and Clinical Excellence. Costing statement: 'Depression: the treatment and management of depression in adults (update)' and 'Depression in adults with a chronic physical health problem: treatment and management'. NICE, 2009 (http://www.nice.org.uk/nicemedia/ live/12329/45943/45943.pdf).

3 Trivedi MH, Rush AJ, Wisniewski SR, Nierenberg AA, Warden D, Ritz L, et al. Evaluation of outcomes with citalopram for depression using measurementbased care in $\mathrm{STAR}^{\star} \mathrm{D}$ : implications for clinical practice. Am J Psychiatry 2006; 163: $28-40$

4 Fostick L, Silberman A, Beckman M, Spivak B, Amital D. The economic impact of depression: resistance or severity? Eur Neuropsychopharmacol 2010; 20: 671-5.

5 Gibson TB, Jing Y, Smith Carls G, Kim E, Bagalman JE, Burton WN, et al. Cost burden of treatment resistance in patients with depression. Am J Manag Care 2010; 16: 370-7.

6 Russell JM, Hawkins K, Ozminkowski RJ, Orsini L, Crown WH, Kennedy S, et al. The cost consequences of treatment-resistant depression. J Clin Psychiatry 2004; 65: 341-7. 
7 Greenberg P, Corey-Lisle PK, Birnbaum H, Marynchenko M, Claxton A. Economic implications of treatment-resistant depression among employees. Pharmacoeconomics 2004; 22: 363-73.

8 Mccrone $\mathrm{P}$, Knapp $\mathrm{M}$, Kennedy $\mathrm{T}$, Seed $\mathrm{P}$, Jones $\mathrm{R}$, Darnley $\mathrm{S}$, et al. Cost-effectiveness of cognitive behaviour therapy in addition to mebeverine for irritable bowel syndrome. Eur J Gastroenterol Hepatol 2008; 20: 255-63.

9 Lam DH, McCrone P, Wright K, Kerr N. Cost-effectiveness of relapseprevention cognitive therapy for bipolar disorder: 30-month study. Br J Psychiatry 2005; 186: 500-6.

10 Mccrone P, Ridsdale L, Darbishire L, Seed P. Cost effectiveness of cognitive behavioural therapy, graded exercise and usual care for patients with chronic fatigue in primary care. Psychol Med 2004; 34: 991-9.

11 Mccrone P, Knapp M, Proudfoot J, Ryden C, Cavanagh K, Shapiro DA, et al. Cost-effectiveness of computerised cognitive-behavioural therapy for anxiety and depression in primary care: randomised controlled trial. $\mathrm{Br} J$ Psychiatry 2004; 185: 55-62.

12 Hollinghurst S, Peters TJ, Kaur S, Wiles N, Lewis G, Kessler D, et al. Cost-effectiveness of therapist-delivered online cognitive-behavioural therapy for depression: randomised controlled trial. Br J Psychiatry 2010 197: 297-304.

13 Thomas L, Abel A, Ridgway N, Peters TJ, Kessler D, Hollinghurst S, et al. Cognitive behavioural therapy as an adjunct to pharmacotherapy for treatment resistant depression in primary care: the CoBalT randomised controlled trial protocol. Contemp Clin Trials 2012; 33: 312-9.

14 Wiles N, Thomas L, Abel A, Ridgway N, Turner N, Campbell J, et al. Cognitive behavioural therapy as an adjunct to pharmacotherapy for primary care based patients with treatment resistant depression: results of the CoBalT randomised controlled trial. Lancet 2013; 381: 375-84.

15 National Institute for Health and Clinical Excellence. Guide to the Methods of Technology Appraisal 2008. NICE, 2008.

16 Beck A, Steer R, Brown G. Beck Depression Inventory: Manual (2nd edn). The Psychological Corporation, 1996.

17 Lewis G, Pelosi AJ, Araya R, Dunn G. Measuring psychiatric disorder in the community: a standardized assessment for use by lay interviewers. Psychol Med 1992; 22: 465-86.

18 Lewis G. Assessing psychiatric disorder with a human interview or a computer. J Epidemiol Community Health 1994; 48: 207-10.

19 Beck A, Rush AJ, Shaw B, Emery G. Cognitive Therapy: Basics and Beyond. Guilford Press, 1995

20 Beck A, Rush AJ, Shaw B, Emery G. Cognitive Therapy of Depression. Wiley, 1979

21 Moore RG, Garland A. Cognitive Therapy for Chronic and Persistent Depression. John Wiley \& Sons, 2003.

22 Ware J-El, Kosinski M, Keller SD. A 12-item Short-Form Health Survey: construction of scales and preliminary tests of reliability and validity. Med Care 1996; 34: 220-33.

23 Brooks R. EuroQol: the current state of play. Health Policy 1996; 37: 53-72.

24 Seivewright $H$, Green J, Salkovskis $P$, Barrett B, Nur U, Tyrer $P$. Cognitive-behavioural therapy for health anxiety in a genitourinary medicine clinic: randomised controlled trial. Br J Psychiatry 2008; 193 332-7.

25 Curtis L. Unit Costs of Health and Social Care 2010. PSSRU, 2011.

26 Munro J, Nicholl J, O'Cathain A, Knowles E, Morgan A. Evaluation of NHS Direct First Wave Sites: Final Report of the Phase 1 Research. University of Sheffield, 2001.

27 Heaney D, O'Donnell C, Wood A, Myles S, Abbotts J, Haddow G, et al. Evaluation of the Introduction of NHS 24 in Scotland. University of Aberdeen, 2005

28 Department of Health. 2010-11 Reference Costs Publication. Department of Health, 2011.

29 British Medical Association, Royal Pharmaceutical Society of Great Britain. British National Formulary 59. British Medical Association and Royal Pharmaceutical Society of Great Britain, 2010.
30 The Automobile Association. The AA Schedule of Motoring Costs. (http:// www.theaa.com/motoring_advice/running_costs/advice_rcosts_guide.html).

31 Office for National Statistics. 2010 Annual Survey of Hours and Earnings. ONS, 2011.

32 Dolan P, Gudex C, Kind P, Williams A. A Social Tariff for EuroQol: Results from a UK General Population Survey. Univeristy of York Working Paper 138. Centre for Health Economics, University of York, 1995.

33 Manca A, Hawkins N, Sculpher M. Estimating mean QALYS in trial-based cost-effectiveness analysis: the importance of controlling for baseline utility. Health Econ 2005; 14: 487-96.

34 van Buuren S, Boshuizen HC, Knook DL. Multiple imputation of missing blood pressure covariates in survival analysis. Stat Med 1999; 18: 681-94.

35 Department of Health. Improving Access to Psychological Therapies. Department of Health, 2012 (http://www.iapt.nhs.uk/about-iapt/).

36 Brazier, J. Measuring and valuing mental health for use in economic evaluation. J Health Serv Res Policy 2008; 13: 70-5.

37 University of Sheffield. SF-6D. University of Sheffield 2012 (http:// www.shef.ac.uk/scharr/sections/heds/mvh/sf-6d).

38 Wiles N, Hollinghurst S, Mason V, Musa M, Burt V, Hyde J, et al. A randomised controlled trial of cognitive behavioural therapy as an adjunct to pharmacotherapy in primary care based patients with treatment resistant depression: a pilot study. Behav Cogn Psychother 2008; 36: 21-33.

39 Davis JC, Liu-Ambrose T, Khan KM, Robertson MC, Marra CA. SF-6D and EQ-5D result in widely divergent incremental cost-effectiveness ratios in a clinical trial of older women: implications for health policy decisions. Osteoporos Int 2012; 23: 1849-57.

40 Barton GR, Sach TH, Avery AJ, Jenkinson C, Doherty M, Whynes DK, et al. A comparison of the performance of the EQ-5D and SF-6D for individuals aged $\geqslant 45$ years. Health Econ 2008; 17: 815-32.

41 Brazier J, Roberts J, Tsuchiya A, Busschbach J. A comparison of the EQ-5D and SF-6D across seven patient groups. Health Econ 2004; 13: 873-84.

42 Longworth L, Stirling B. An empirical comparison of EQ-5D and SF-6D in liver transplant patients. Health Econ 2003; 12: 1061-7.

43 Lamers LM, Bouwmans CAM, van Straten A, Donker MCH, Hakkaart L. Comparison of EQ-5D and SF-6D utilities in mental health patients. Health Econ 2006; 15: 1229-36.

44 Rodger M, Asaria M, Walker S, McMilln D, Lucock M, Harden M, et al. The clinical effectiveness and cost-effectiveness of low-intensity psychological interventions for the secondary prevention of relapse after depression: a systematic review. Health Technology Assess 2012; 28: 1-130.

45 Claxton K, Martin S, Soares M, Rice N, Spackman E, Hinde S, et al. Methods for the Estimation of the NICE Cost-Effectiveness Threshold. Research Paper 81. Centre for Health Economics, University of York, 2013.

46 Department of Health. The GP Patient Survey. NHS England, 2012 (http:// www.gp-patient.co.uk)

47 Kendrick T, Chatwin J, Dowrick C, Tylee A, Morriss R, Peveler R, et al. Randomised controlled trial to determine the clinical effectiveness and costeffectiveness of selective serotonin reuptake inhibitors plus supportive care, versus supportive care alone, for mild to moderate depression with somatic symptoms in primary care: the THREAD study. Health Technol Assess 2009; 22: $47-56$.

48 Mukuria C, Brazier J, Barkham M, Connell J, Hardy G, Hutten R, et al. Cost-effectiveness of an Improving Access to Psychological Therapies service. Br J Psychiatry 2013; 202: 220-7.

49 Ivanova J, Birnbaum $\mathrm{H}$, Kidolezi $\mathrm{Y}$, Subramanian $\mathrm{G}$, Khan S, Stensland M. Direct and indirect costs of employees with treatment-resistant and nontreatment-resistant major depressive disorder. Curr Med Res Opin 2010; 26 2475-84.

50 Layard R, Banerjee S, Bell S, Clark DM, Field S, Knapp M. How Mental Illness Loses Out in the NHS. Centre for Economic Performance, London School of Economics and Political Science, 2012.

51 Black C, Frost D. Health at Work - An Independent Review of Sickness Absence. TSO (The Stationery Office), 2011. 